\title{
Epistemology of experience in E. Husserl's phenomenology and Dewey's instrumentalism
}

\author{
Evgeniy Plekhanov ${ }^{1}$ and Elena Rogacheva ${ }^{2}$ \\ ${ }^{1}$ Vladimir Branch of Russian Presidential Academy of National Economy and Public \\ Administration, 600017, Russia \\ ${ }^{2}$ Vladimir State University, 600017, Vladimir, Russia
}

\begin{abstract}
The themes present in the philosophy of E. Husserl and J. Dewey could hardly lie outside the interest of considering the major contribution both made to Twentieth-century philosophy and intellectual culture. Comparison of their philosophical positions is carried out, as a rule, on the basis of contrasting transcendentalism and empiricism. Unlike the established tradition, the authors of the article draw attention to the substantial commonality of phenomenology and instrumentalism, thematically conditioned by the founders of these teachings working out the problems of the existential nature of human experience, its horizontal structure and semantic context of the life world. Analysis of the epistemological reflection of the concept of "experience" in the teachings of Husserl and Dewey allowed to show, that their positions do not exclude, but complement each other. The paper is devoted to the 160 th Anniversary of John Dewey's Birth (1859-1952), that would be widely celebrated not only in USA and Europe but in Russia, Japan, China and Latin America.
\end{abstract}

The basis for such a proposal is the similarity of the central theme that lies at the core of Husserl and Dewey's intellectual efforts, namely the theme of the future.

The main reasons for Husserl to turn to the problems of transcendental phenomenology characterized by the analytics of "historical teleology of the infinite aim of the human intellect" was the crisis of Western Culture and anxiety about the future of European humankind. The essence of the crisis for scientific civilization at its height is vividly seen in the loss of the practical sense of life by human beings bent on conquering the world. The contradiction between "life self-excitement" on the part of a human being and a scientific way of his/her self-determination in the world reached at the beginning of the Twentieth century its highest degree of acuteness.

Husserl thought the search for a new liveliness and spirit that would provide a great, if distant, future of mankind to be a positive alternative to that "European existence" decay, as he proclaimed the immortality of the spirit [1].

As for Dewey, the theme of the future that is open and initially without guarantee that required constant and persistent human effort was the focus of attention. He believed that

* Corresponding author: eaplekhanov@gmail.com 
with the establishment of democracy there appeared an entirely new mode of social life and activity based not on tradition and dogmatic authority but on critical reflection and creative problem solving. Democracy for him meant a person brings into his life-world a unique notion of freedom that at the same time sets problems for his/her personal future.

Dewey stressed the idea that "...we live in a dynamic, developing world. So he considered the main task for humanity to be concentrated not on revisiting the past but on reconstructing the future. For Dewey the past (all our knowledge in comparison to thinking lies in the past) has its value only if it is capable of providing validity and effectiveness in our future decisions and actions" [2].

The evaluation of the crisis of Western culture is different for Dewey and Husserl. For the leader of the instrumentalism, the development of democracy is a phenomenon of a permanent crisis. Democracy can function only as a process, as a constant permanent transcending of social experience and overcoming of its marked life-world borders. In this sense, the fact of the crisis of democracy is less important than the openness of democracy to the horizon of infinite goals, tasks and needs. Husserl sees in the crisis of European spirit not the stage of Faust's daring but Wagner's tiredness and disillusionment with a newEuropean philosophical and scientific way of cognition.

In spite of the marked difference, both the scientists' display similarity in giving importance to life-sense and rational-reflective components of human experience for the future of democracy and culture.

Considering the place that the category of "experience" takes in the phenomenology of Husserl and the instrumentalism of Dewey, we may consider a creative work of both thinkers as an attempt to give way to the "philosophy of experience".

For Dewey, the philosophy in question is, to paraphrase the saying of Lincoln about democracy, one of, by, and for experience. No one of these words; if, by or for, names anything which is self-evident. Each of them is a challenge to discover and put into operation a principle of order and organization which follows from understanding what educative experience signifies [3].

We would stress that Husserl and Dewey were far from identifying experience with reality of individual mental life. For Husserl this statement needs no special confirmation. As for transcendental-phenomenological sense of the notion "experience" in the philosophy of instrumentalism, it is not that evident. One should pay attention to the declaration of Dewey to create "a scientific philosophy of Experience". Dewey stressed:

"The organic connection between education and personal experience; or that the new philosophy of education is committed to some kind of empirical and experimental philosophy. But experience and experiment are not self-explanatory ideas. Rather, their meaning is part of the problem to be explored. To know the meaning of empiricism we need to understand what experience is [4].

Dewey's intention was in tune with Husserl's but their realization of this task diverse in important ways. In his theory of experience Husserl started from the theory of intentionality, that made possible for him to avoid a dualism between the quality of an object and sensation, a thing and an image, physical and mental processes. Intentional objects are not the reflection of material phenomena and objects. They are constituted by mechanisms of functioning of consciousness and are given to us in the form of internal experience, the modes of which (presentation, perception, imagination, desire, evaluation \& others) express a process-dynamic character of a mental reality. Therefore, from the point of phenomenology it is more important to know how immanent objects are given to us than what is represented in our emotional or cognitive experience.

For Husserl, every attempt to put physical objects under mental phenomena blocks the singling out of experience from natural things and processes. Phenomenological reduction is a necessary prerequisite that makes possible for a reflective consciousness to face itself 
as an object for description and analysis. At the same time, reduction is something more than just a pure methodical procedure not concerning ontological status of external world and the being of consciousness.

As contemporary researchers mark "...what people call a real world, what physicists call transcendental substances, - all of it, according to Husserl, is nothing more but correlates of consciousness, having partly a mythological character" [5].

If we look at the world of nature in the natural attitude of our consciousness then it will lead to the conclusion that the intentional content of experience needs no external reality. On the contrary, world may be understood as reality only in our emotional experience.

As Husserl states: "Consciousness if it is understood "in its pureness" must be accepted as a closed interlink of being, and namely as an interlink of absolute being, where nothing can penetrate into and nothing can escape from... On the other side all space-temporary world, to which a human being, a human "self" as main subordinate realities belong- is in its sense only intentional being, the being that has only a relative, secondary sense of being for consciousness... The being of this type is an identity of uncontradictory motivated varieties of experience and it is open to contemplation and determination- above of all this such a being is just nothing, or, to be more exact, it is the being, for which every above this is an antisensible thought" [6].

Philosophical radicalism of Husserl, removing ontological dualism of consciousness and world by means of objective existence content to intentional experience existence was not typical for Dewey. The limitation of experience by consciousness frames was considered by Dewey as one-sided European rationalism sign fighting fruitlessly for many centuries with one-sided empiricism. He also considered the opposition of real and ideal that western philosophy proclaimed not valid.

"Division of world in two aspects of being, one of which is the highest, accessible only to reason and ideal in gyst, and the other - the lowest, material, changeable, empirical, accessible only to sense observation, inevitably turns to the idea that knowledge is in its essence contemplative" [7]. Thinking of cognition as a passive perception, intellectual perception is not only one of philosophical mistake; it has a far going consequences in the field of actual relations of the human being and the world. It is formed on the basis of corresponding to historical experience of relations with the world. As soon as thanks to experimental natural sciences it becomes clear that cognition is active and operative, all antinomies of ideal and real, consciousness and reality are removed. Loosing transcendental character, the kingdom of absolute and reasonable becomes the horizon of possibilities, means and ways of accomplishing practical aims and tasks. So, if Husserl tried to eliminate natural setting as a source of undesirable naturalism, for Dewey external reality, the outside world was a necessary condition for cognition as active action that allows practically to transform reality, giving it the form of our consciousness.

Hence, "the main function of philosophy is rationalization of the experience possibilities, especially, as Dewey stressed, collective human experience " [8].

Interpretation of reason in Dewey's philosophy as "experimental intellect" does not mean that experience in itself has no principles of connection and organization.

So it is necessary to clarify what is the constitutive structure of experience in phenomenological and pragmatic contexts. It is necessary to analyze the contribution of Husserl to dynamically processed understanding of the experience of consciousness. From the phenomenological concept of the consciousness of experience is seen as a permanent process of changing emotional experiences. Only in permanent constituting of "pure" experience consciousness finds the reality of its being. The movement of intentional objects is to some extent an immanent way of life accomplishment of our consciousness.

"The life of consciousness is a current, and every cogito is fluctuating, it has no fixed elements and relations"[9]. 
Though as Husserl had warned, the image of the current is very treacherous, as it is easily associated with concrete being-becoming, and so, opens the perspective of the way of theoretical thought to relativism and subjectivity, that the founder of phenomenology opposed that much. Therefore, in another line of his writings we find a special explanation related to the fact that dynamism of life of consciousness is a product of incompleteness of a row of reductions.

Thanks to phenomenological epoche, experience is shown in a form of different "blinks", psychological phenomena - feelings, emotions, thoughts, excitements, fantasies etc. Husserl distinguishes between the "natural attitude", that means our straightforward involvement with things and the world, and the "phenomenological attitude", the way we carry our philosophical analysis of the intentions exercised in the natural attitude and the objective correlates of these intentions. In phenomenological attitude a human being takes a distance from actions and intentions and convictions of the natural attitude and contemplates their structure. Husserl calls this suspension the phenomenological epoche. But it does not mean that the person doubts or negates all the intentions, convictions.

"In our human life we begin, of course, in the natural attitude, and the name for the process by which we move to the phenomenological attitude is called the phenomenological reduction, a "leading back" from natural beliefs to the reflective consideration of intentions and their objects" [10].

These uncertainties form an empirical level of experience. So phenomenological psychology is possible owing to eidetic reduction that reveals a deeper level of internal experience, a level of invariant structures of emotional experiences. In phenomenological attitude we look at the intentions that we normally look through, those that function anonymously in our straightforward involvement with the world.

"In a current, - as Husserl stressed, - there rules a very well expressed technique" [11].

A deep layer of experience, formed by stable configurations of intentional objects, cannot be seen as a unity and division of separate elements of consciousness. A concrete emotional experience is a sort of a chain in the continuous chain of other emotional experiences that form a permanent meaning field of our experience. At the same time, for Husserl, emotional experience is the unity of immanent running, where one can see different phases and intervals:

In every partial perception running as a phase of integrated perception, the whole meaning of the object that is percepted is seen in every phase meaning, it is fed by phase meanings, becoming deeper and being determined more in detail, but it is by no means integrated from separate parts and united in a whole one with the help of gestalt sensitivity [12].

Husserl's analysis of the constitution of integrated process of experience meant to critique the kind of associationism that still rules in psychology and that attempts to explain the integrity of internal experience by contingent links disconnected from the context of action. It is also a critique of reductionism in natural science that seeks to explain the content of psychic reality based on the activity of the perceptive mechanisms of the sense organs. Integrity and continuity of internal experience is supplemented by its capacity to permit "soft" and flexible self-determination. The current of consciousness includes an immanent teleology as the constructive basis for the collection of discrete, locally modal, temporal, and emotional experiences into an integrated unity having the characteristics of a meaning configuration.

The configuration's state is explained by the presence of some definite internal form in a permanent field of experience. This form gives integrated outlines (contours) to its intentional content. The capacity of consciousness to make distinct outlines of the objects of internal experience is accomplished in noemic-poetic structure of intentionality. Noemic-poetic unity is of a projective character. It is explained by the fact that intentional 
objects in opposition to physical objects, belong to the field of human experience and are projected by constitutive capacities of our consciousness. Besides, being definite they are not schematic, but variable.

Owing to this there takes place what Husserl called "attentional shifts in noemic and noetic aspect" of emotional experience. The possibility of existence of every meaningful concrete integrity in different modes (in forms of perceptible, imaginative, desirable, thinking \& etc.) is characterized by them as intentional projects, as various variants of one and the same meaning of the object.

The notion of "intentional projects" presupposes not only a meaningful content of every concrete emotional experience, but also its principal peculiarity as horizontness. Husserl himself uses the term "horizon" at least in three different meanings.

Firstly, by horizon Husserl means a potential level of experience in opposition to actual, completed state. As every emotional experience includes a sort of intrinsic potentiality it cannot be seen as completely realized in none of its states. In this case" horizon is an internal logic, that determines the perspectives of the perception unfolding and it's striving to exhaustive accomplishment.

Sartre explains the ex-statics of this kind, typical of human reality, by the need of consciousness to fill a gap in its ontological insufficiency. Therefore, the horizon of "being for oneself" is dependent on projections of our desires, in the form of which the intuitive striving to fullness and completeness of our emotional experiences is given to us [13]. In connection to this, actual acts of consciousness potentially contain both possible and previous experience of excitements, they are accomplished in a permanent horizon of immanent time and so can be unfolded as perspective and retrospective projects.

Perspective projects are protentions that are going to enter our perception and to be accomplished in intentions of expected, desired, anticipated, foreseen... Retrospective horizontness is not equal to earlier accomplished intentional acts. Otherwise, it would loose its projective character. The horizon of the past is displayed through intentionality of recollections, each of which takes us to a permanent infinity of other recollections. And retaintional projects are always not a reconstruction of previous perceptions but its new excitement, comprehension, explanation, evaluation as the past always appears to us in unprocessed and unexpected image [14].

The unity of relational and potential projectivity may be seen as a two-faced Janus. It forms a diachronic horizon of constitutive experience of the activity of our consciousness.

Secondly, the notion of horizon is connected by Husserl with potential completeness of the content of actually given experience. Every initial perception (image, knowledge, or meaning) is impoverished, schematized but at the same time, it has a capacity to complete, to enrich a content, to find out something hidden. In the process of intentional perceptive acts there occurs a change of various parameters that determine an object in experience (distance, background, a degree of lighting etc.). So, possible variations of perception of this object form what is called a synchronic horizon of a current of consciousness. In "transverse" slit intentional experience may be seen as a total combination of inter-linked projects (or variants) of intentional objects potentially contained in an actual content of emotional experiences.

Finally, an environment of internal experience gives the horizon. Every concrete perception is born and grows within the frame of other perceptions that owing to synthetic capacity of consciousness flow together into a permanent universal cognitive field. Therefore, a general emotional background, unaware settings of consciousness, rational and axiological dominants create a contextual horizon of emotional experience. If a surface noemic-noetic structure of an object is fairly determined and factual, then its deeper , meaningful structure is variable, as it gives a chance to different interpretations. James 
meant this very circumstance when he analyzed a horizontal organization of sense perception.

"The meaning, the value of an image is mainly in its addition, its light shadow (penumbra) of the elements of thought surrounding and accompanying it. Better to say, this penumbra together with this image makes the whole... leaving the very image the same as it was before. It gives it a new function and a fresh color" [15].

The aforementioned properties of intentional life of experience are not exhaustive but sufficient to have a notion about Husserl's understanding of experience.

Dewey as well as Husserl admitted dynamic-process understanding of consciousness.

" The processes of adaptive actions, habits, active functions, links and emotions, sensemotor co-ordinations are considered to be real experience "current" [16].

Though in opposition to Husserl, his strivings were focused mainly on research of elaborating and accumulating of a subject in the process of active environment mastering but not constituting and organization of internal experience. It is well known that Dewey names active and standard components of experience. Active side of experience is seen in actions with the objects and so is of intentionally practical character. The passive side is in back reaction of the world of objects on us. In making links between our influences and counteractions of things is the essence of experience, its content richness.

So, Dewey stresses: "the main in experience is the combination of activity and passivity, but not in its cognitive aspect...Experience turns to be cognitive only as it is accumulative, goes to something, has a sense" [17].

J. Harrison and I.E. Shargel are right, stressing that Husserl focuses his attention on cognitive aspect, while Dewey does on manipulative aspect of experience.

"Now we see, they conclude, - essential differencies, that are hidden in the basis of conceptual agreement"[18].

In comparison to Husserl, Dewey pays more attention to elaboration and growing of experience on the part of a subject and less focuses on constituting and organization of internal experience. So the experience may be seen as consisting of active and standard components. An active side of experience means that it presupposes an action with objects; so, it presupposes intentionality in a sense of directness, selectivity of influences on an object. The passive side is in reverse influence of world objects on human beings. The sense of experience is in making connections between our influences and counteractions of things.

Dewey stressed, that "the main thing in experience is the combination of activity and passivity, and not its cognitive aspect" [19].

At first sight, this interpretation of the nature of experience does not open a wide space for its critique from the positions of "pure phenomenology". In reality, it is not so.

First, we should stress that Dewey very clearly says that the relations between the objects cannot be understood outside the experience context. More to it, noemic content of things can be seen only within a structure of intentional relations, A thing may be done in experience as hard or soft, colored or colorless, useful or not only if it is in relation with us. Secondly, Dewey gives priority to active elements of experience and not to the cognitive, but that does not mean he underestimates the importance of rational reflection in the process of consolidating the experience, including its intentionality and projectivity.

"Thinking, as he points, is an explicit expression of intellectual component of our experience. Owing to thinking, an aimed action becomes possible" [20].

In its turn, the action having a conscious aim is based not on a psychological but on a transcendental level of experience.

Moreover, thirdly, Dewey has not a transcendental level in the sense of constitutive structures of consciousness in Kant or Husserl's sense, though we bring prior learning, especially habits, to experience. As for Husserl, his attitude towards natural settings 
undergoes a great evolution that may be explained by his dealing with the problem of intersubjectivity and "life-world". To be more exact, Husserl does not simply accept and rehabilitate a natural assumption, but analyzes it as the dominant state of human experience by means of phenomenology. The product of it is the notion "life-world" as a field of cultural and historical experience that is seen as the content for collective practice. In its horizon all possible meanings, concepts, norms, and values of human life lie. So, Husserl removes some duality in Dewey's understanding of the world of objects, trying to underline that every concrete person deals in his experience not with the things and phenomena transcendental to his consciousness, but with the fragments and regions of cultural and historic life-world.

Dewey wrote that, "... we live from birth to death in a world of persons and things which in large measure is what it is because of what has been done and transmitted from previous human activities. When this fact is ignored, experience is treated as if it were something that goes on exclusively inside an individual's body and mind. It ought not to be necessary to say that experience does not occur in a vacuum. There some sources outside an individual which give rise to experience. It is constantly fed from its springs" [21].

It is here that we can sum up the results of our comparison and contrast. The interpretation of experience by Husserl and Dewey is complimentary, at least, in two aspects. Dewey adds to the Husserl's discovery of the horizontal structure of experience a vertical structure, that is seen as opening up to consistent stages of potentially endless growth. As for Husserl, systematically clarifying a transcendental-intentional content of life-world he adds much to the theory of activity that Dewey had put forward. Husserl binds the gaining of individual experience with a cultural-historical context.

\section{References}

1. E. Husserl, Voprosi filosofii, 3, 115 (1986)

2. J. Dewey, Democracy and Education, 145, (2000)

3. J. Dewey, Experience and Education, 29, (1938)

4. J. Dewey, Experience and Education, 25, (1938)

5. The Outlines of Phenomenological Philosophy, 14 (1997).

6. E. Husserl, The Ideas Towards Pure Phenomenology, 9 (1994)

7. J. Dewey, Reconstruction in philosophy. Human problems, 84 (2003)

8. J. Dewey, Reconstruction in philosophy. Human problems, 85 (2003)

9. E. Husserl, Logos, 2, 17 (1991)

10. E. Husserl, The Cambridge Dictionary of Philosophy, second edition. 405 (1999)

11. E. Husserl, Logos, 2, 17 (1991)

12. E. Husserl, Logos, 3, 74 (1992)

13. J.P. Sartre, Being and Nothingness: The Experience of Phenomenological Ontology, 118-123 (2000)

14. E. Husserl, Phenomenology of Internal Consciousness of Time, 1, 32-67 (1994)

15. W. James, Psychology, 71 (1991)

16. J. Dewey, Reconstruction in philosophy. Human problems, 69 (2003)

17. J. Dewey, Democracy and Education, 134 (2000)

18. J.W. Garrison, E.I. Shargel, Educational Theory, 38, 246 (1988)

19. J. Dewey, Democracy and Education, 134 (2000) 
20. J. Dewey, Democracy and Education, 139 (2000)

21. J. Dewey, Experience and Education, 39-40 (1938) 